\title{
USE OF RESOURCE BLOCKS IN SLAM II FOR THE EVALUATION OF OPERATOR-MACHINE ASSIGNMENTS
}

\author{
Lynn S. Lachenmaier \\ Jeffery K. Cochran \\ Department of Industrial and Management Systems Engineering \\ Arizona State University \\ Tempe, AZ 85287-5906, U.S.A.
}

\begin{abstract}
This paper examines the problem of assigning jobs to a set of manufacturing machine operators. This classic problem involves a set of semi-skilled personnel who can run subsets of machinery. The number of operators assigned to groups of machinery can be varied to optimize throughput. While the operator-machine problem has been studied in previous literature using a wide variety of analytical algorithms, we look at a solution that uses the RESOURCE block in SLAM II to simulate possible operator-machine combinations. A semiconductor fabrication facility was modeled using the operators as resources, and four alternate assignments were compared. Our concept for developing a simulation model of this problem in SLAM II proved to be an effective way to evaluate operator-machine assignments.
\end{abstract}

\section{INTRODUCTION}

A classic problem for manufacturing managers is the operator-machine assignment problem. This problem arises when there are more machines than operators, and each operator has been trained on only a subset of the available machines (Wittrock 1992). One operator can run more than one machine, and conversely, multiple operators can share the duties of running one machine or machine group. If an operator is assigned to too many machines, parts sit idle in one machine while the operator is busy with the others. This "machine interference" affects the overall system output (Stafford 1988).

In many facilities, there is no structured method of choosing the operator-machine assignment. The decision is typically left to the production supervisor and can vary from shift to shift within the same department. Decisions are made based on experience or informal rules of thumb (Jackman and Johnson 1993). This lack of structure can lead to inefficient combinations of operators and machines restricting the potential output of the facility.

This paper examines prior approaches to this problem and explores the possibility of comparing operator-machine assignments that have been determined acceptable to management as design questions in a SLAM II simulation model. The operators are modeled as resources in a SLAM II RESOURCE block. Four assignments from a semiconductor fabrication facility at Delco Electronics in Kokomo, IN are compared to illustrate the application of this concept.

\section{BACKGROUND}

Many approaches have been proposed to determine operator assignments that optimize system output. One method discussed in the literature is to create a mathematical representation of the system. Stafford (1988) shows how deterministic mathematical models of the operator-machine assignment problem can be solved both graphically and through the calculation of three relatively simple formulas. Using this method provides the manager with " $\mathrm{K}$," the optimum number of machines to assign to any one operator. The formulas are easily computed, but assuming manufacturing processes to be deterministic limits the applications of this approach. Stafford's formulations also assume that all operators will be assigned the same number of machines.

More flexibility and realism are gained by modeling the system as probabilistic. Wittrock (1988) demonstrates the problem as a network flow problem. His more complex model deals with the concept that operators will have overlapping skill sets. The optimum number of jobs is not found per operator, but for teams of operators with identical skills. This approach finds an 
optimum number of operators to assign to a job, but this technique requires complex mathematical algorithms to maintain the robustness of its solutions. Queueing networks are popular in manufacturing applications, but do tend to have difficulty representing systems that involve merging parts together in sub-assemblies, such as the bonding of wafers in multi-stack semiconductor devices (Jackman and Johnson 1993).

Lutz et al. (1994) use a decision support system (DSS) to generate operator assignment schedules by solving a dynamic programming heuristic. However, this method relies on the assumption of a balanced production line (where the average processing times on all machines are the same).

\section{MODELING THE OPERATOR- ASSIGNMENT PROBLEM WITH SLAM II}

Many of the assumptions that are required for the mathematical models are not necessary for a simulation model of a manufacturing system. Operators, or servers, do not have to be assigned equal numbers of jobs. All machines can have probabilistic processing times with totally different underlying distributions. Merging subassemblies is easily done.

The operator-machine assignment problem can be tackled in the simulation language SLAM II by making use of the RESOURCE block and the AWAIT node (Pritsker 1995). Just as a machine can be a resource for which parts wait, the operators can also be coded as resources for waiting parts. Machine interference, then, is represented by parts sitting in the AWAIT node after a machine has become available while all the operators assigned to that machine are utilized elsewhere. Different design alternatives can then be tested by altering the operator RESOURCE block and changing the resource label (RLBL) specified in the AWAIT and FREE nodes.

This method does not aim to optimize the combination of operators and machines, but instead allows management to compare the relative effectiveness of assignments known to be acceptable under other constraints, such as cross-training, union rules, or operator limitations. The simulation model acts a source of feedback to the manager who wants to know "which of these several assignment combinations is best," without disrupting his personnel by trying them all in the real factory.

While this paper focuses on the methods used to represent operator assignment in SLAM II, other simulation packages also allow the user to specify which machines an operator can attend. MANSIM, for example, which does not require the level of programming of SLAM II, allows the user to input "operator skills" in a Windows menu format. The same goal of creating machine interference is accomplished.

\subsection{System Description}

This approach was tested on a real operator-machine assignment scenario at Delco Electronics. A fairly complex model was designed that captured the essential functions of an acid etch room in a semiconductor fab. The model demonstrated the processing of five part types through seventeen major operations. Two part types were sub-assemblies that are bonded to two of the other three parts. The seventeen operations consisted of twenty-eight individual machines. Eighteen operators were available to run jobs.

The operators were modeled in the RESOURCE block by listing the AWAIT nodes associated with a coded group of one or more operators (see Table 1). The groups were identified as "OPERA, OPERB," etc. For instance, to show that two operators (grouped as OPERA) would be assigned to jobs consisting of machines 1,4 and 8, the RESOURCE code would look like "RESOURCE/OPERA(2), 1,4,8."

Table 1: A SLAM II RESOURCE Block that Defines the Operator-Machine Assignments for One Possible Arrangement

\begin{tabular}{|l|r|r|r|r|r|r|}
\hline OPERA & 1 & 2 & 11 & & & \\
\hline OPERB & 2 & 5 & 66 & 23 & 55 & 7 \\
\hline OPERC & 1 & 9 & 17 & 59 & 63 & \\
\hline OPERD & 1 & 25 & & & & \\
\hline OPERE & 1 & 53 & & & & \\
\hline OPERF & 1 & 51 & 57 & 61 & 15 & \\
\hline OPERG & 1 & 19 & & & & \\
\hline OPERH & 1 & 33 & 43 & & & \\
\hline OPERI & 1 & 35 & 45 & & & \\
\hline OPERJ & 1 & 37 & 29 & & & \\
\hline OPERK & 3 & 39 & 47 & & & \\
\hline OPERL & 1 & 27 & 65 & & & \\
\hline OPERM & 1 & 21 & & & & \\
\hline OPERN & 2 & 49 & & & & \\
\hline
\end{tabular}

In this way, any number of operators can be associated with any number of specific machines. Sharing of jobs is allowed; multiple jobs per operator are allowed. Operators can have different skill sets (the machines to which they could be assigned are limited), or they could all be identical and assignable to any machine.

The entities in the SLAM II model are lots of wafers that need processing through the seventeen workstations of the acid etch room. The product flows are modeled very simply by a series of AWAIT and FREE nodes (see Figure 1). When a part arrives at a workstation, it waits first for a machine to be available, 
then it waits for an operator from the group assigned to that job to become available. When the operator finishes her setup and measurements, she is freed, and in some cases, the machine runs for some time by itself. Machines that do not run without the full attention of the operator are modeled with a zero duration activity between the operator free and the machine free. Finally the machine is freed and the part proceeds to the next station. This pattern is repeated thirty times in the model to represent the flow of product through the entire system.

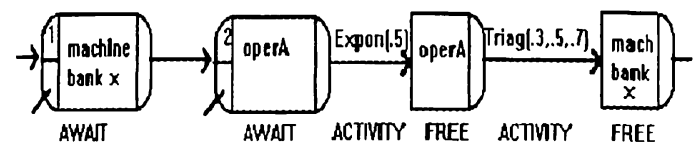

Figure 1: Illustration of a Portion of the Network Diagram Where a Part Arrives to a Given Workstation

Durations for operator time and machining time were derived from data collected by engineering. Raw data was used to fit probability distributions. In this system, different machines have different levels of variability and were described by totally different distributions. The values used are shown in Tables 2 . From these tables, you can see that highly unique systems can be modeled with SLAM II.

Table 2: Probability Distributions Used as Machine Times (time a machine runs without the aid of an operator) and Operator Attention Times

\begin{tabular}{|c|c|c|c|}
\hline MACH & DISTRIBUTION & OPER & DISTRIBUTION \\
\hline AUTO & TRIAG $(.3,5,7)$ & AUTO & EXPON(.471) \\
\hline BAKE & TRIAG $(.167, .5,1)$ & BAKE & EXPON (127) \\
\hline OXIDE & TRIAG $(075, .218, .433)$ & OXIDE & EXPON(.213) \\
\hline STRIP & TRIAG $(1.083,1.083,1.583)$ & STRIP & EXPON(.122) \\
\hline METAL & EXPON $(.042)$ & METAL & EXPON(.531) \\
\hline $\mathrm{CD}$ & 0 & $C D$ & EXPON(5) \\
\hline CONT & 0 & CONT & EXPON (217) \\
\hline PRINT & UNFRM(1.183,1.267) & PRINT & EXPON (.904) \\
\hline PUNCH & 0 & PUNCH & EXPON(4.483) \\
\hline PAD & EXPON (.1) & PAD & EXPON (.151) \\
\hline BOND & UNFRM $(6,1.033)$ & BOND & EXPON(2.392) \\
\hline $\mid \mathrm{IR}$ & 0 & IR & TRIAG $(217,1.1,13.383)$ \\
\hline VIKING1 & 0 & VIKING1 & UNFRM $(4.2,7.6)$ \\
\hline VIKING2 & UNFRM( $833,1.234)$ & VIKING2 & EXPON(1.628) \\
\hline ECE & EXPON(6.501) & ECE & EXPON(2.939) \\
\hline GLASS & UNFRM $(934,1.167)$ & GLASS & EXPON (235) \\
\hline CAV & UNFRM $(2.723,3.133)$ & CAV & EXPON (.342) \\
\hline $\mathrm{KOH}$ & TRIAG $(1.05,1.991,3.717)$ & $\mathrm{KOH}$ & EXPON(.348) \\
\hline OUT & RNORM $(7.3,2.8)$ & & \\
\hline
\end{tabular}

The basic model was validated by conducting a Kolmogorov-Smirnov (K-S) test on the data used and by examining the initial summary reports generated by the system. Idle times of machinery and operators and system output were compared to historical data. While some pieces of the model were more true to life than others, the results maintained the general behavior of the acid etch room.

\subsection{Design Alternatives}

To examine the SLAM II model's use as a method of evaluating operator-machine assignments, four methods of operator assignment were tested. With eighteen operators and twenty-eight machines, more than $4.099 \mathrm{x}$ $10^{31}(18 !(18 !-8 !))$ combinations are possible, even if we ignore job sharing, and restrict the maximum number of machines run by a given operator to two! However, there is no reason for us to examine more than a minuscule fraction of the possible assignments. In a real facility, a manager would probably want to compare his current practices to a handful of hypothetical alternatives suggested to him by his staff that he knows would satisfy his other constraints.

We reproduced this scenario by comparing four assignments. The two assignments used currently by first shift and second shift were compared, as well as two hypothetical arrangements. The first hypothetical assignment is based on raw labor point data; the other assumes full cross-training of the operators. The resource blocks representing each assignment is shown in Table 3.

Table 3: RESOURCE Blocks that Define the AWAIT Nodes (jobs) Assigned to Each Group of Operators
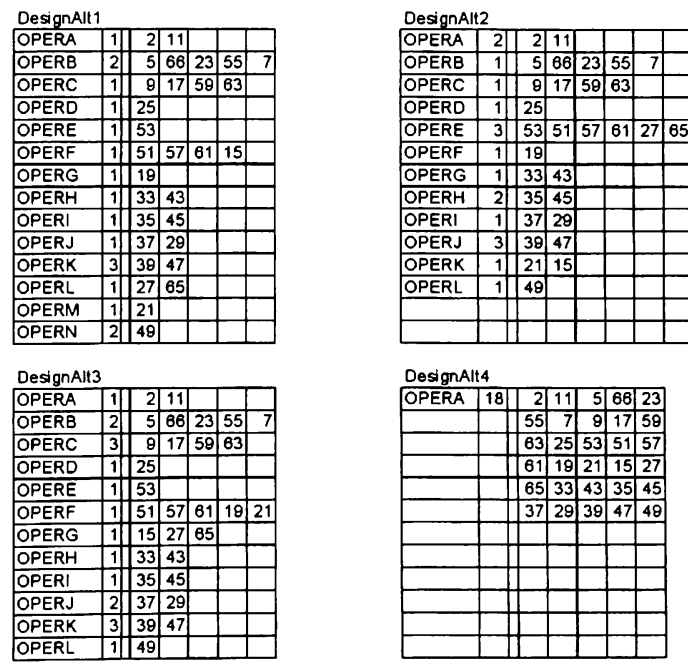

Each alternative was measured by the number of "ships", or total number of lots shipped to the customer from the system over a two week period. Our goal, then was to find the alternative that shipped the most lots. All four designs were compared at their steady state, as data from the warm-up period of the system was truncated and disregarded. Pilot runs of the original 
model were used to determine the proper number of runs to make of each alternative to compare them at a $90 \%$ confidence level within $+/-2$ shipped units. Twenty runs were performed for each design alternative.

The programming time required to test a new arrangement after the base model was established was minimal. From our base model containing 342 lines of code, a new operator arrangement could be created in less than 30 minutes. The models ran in seconds.

\section{ANALYSIS OF RESULTS}

Data was collected from the runs of each design alternative. The maximum number shipped by the system in any two weeks was 39 units and the minimum was 0 . The data for each operator assignment alternative is summarized below in Table 4 .

Table 4: Number of Ships Generated by Each Design Alternative over Twenty Runs

\begin{tabular}{|c|c|c|c|c|}
\hline & DesignAt1 & DesigAt2 & Designtt3 & DesignAt4 \\
\hline \multirow{3}{*}{$n$} & 18 & 10 & 33 & 16 \\
\hline & 15 & 14 & 13 & 14 \\
\hline & 23 & a & 16 & 3 \\
\hline \multirow{3}{*}{$\begin{array}{l}\mathrm{u} \\
\mathrm{m} \\
\mathrm{b}\end{array}$} & 16 & 3 & 19 & 14 \\
\hline & 19 & g & 19 & 18 \\
\hline & 20 & 29 & 15 & 21 \\
\hline \multirow{3}{*}{ e } & 2 & d & 13 & 16 \\
\hline & 11 & 14 & 20 & 16 \\
\hline & 21 & g & 17 & 17 \\
\hline \multirow{3}{*}{$\begin{array}{l}0 \\
f\end{array}$} & 20 & 15 & 18 & 19 \\
\hline & 16 & 1 & 3 & 18 \\
\hline & 21 & 15 & 10 & 12 \\
\hline $\mathbf{s}$ & 16 & 22 & 13 & 20 \\
\hline $\mathrm{h}$ & 17 & q & 13 & 18 \\
\hline \multirow{2}{*}{ i } & 18 & 4 & 3 & 20 \\
\hline & 12 & 8 & 39 & 10 \\
\hline \multirow[t]{4}{*}{ s } & 23 & 26 & 18 & 23 \\
\hline & 13 & 15 & 21 & 22 \\
\hline & 20 & 8 & 19 & 16 \\
\hline & 21 & 2) & 28 & 18 \\
\hline Average & 17.1 & 11.8 & 202 & 166 \\
\hline SdDer & 4.9 & 88 & 80 & 4.5 \\
\hline
\end{tabular}

As you can see, the four design alternatives shipped different numbers of parts. Operator assignment method 3 generated the highest number of ships in a two week period on the average, and therefore could be the best alternative of our four tested. However, since our model is probabilistic and the values vary from run to run, we checked to see if the differences in output between the design alternatives were statistically significant. This was done by analyzing the experiment with Design-Ease software. Design-Ease created the graph shown in Figure 2 to demonstrate the variance in our results.

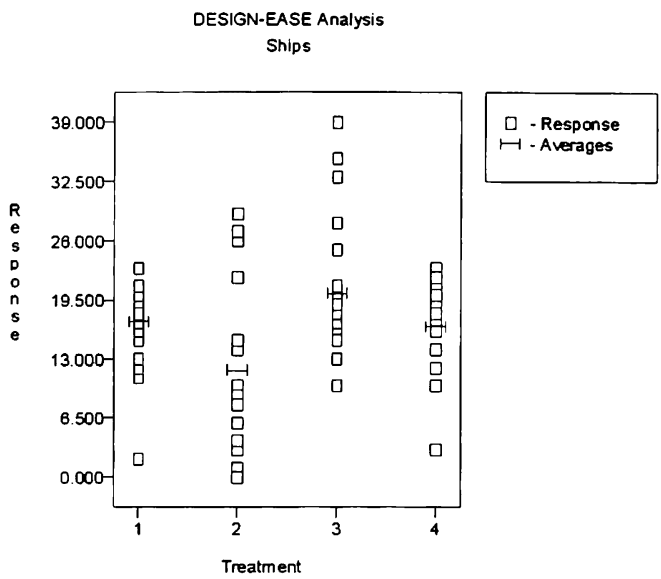

Figure 2: Interpretative Graph from Design-Ease Showing Means and Ranges of the Four Alternatives

An Analysis of Variance (ANOVA) was conducted to determine whether or not the four means were different. The null hypothesis tested was that the four mean ships were equal. The results of the ANOVA are shown in Table 5.

Table 5: ANOVA Results Adapted from Design-Ease

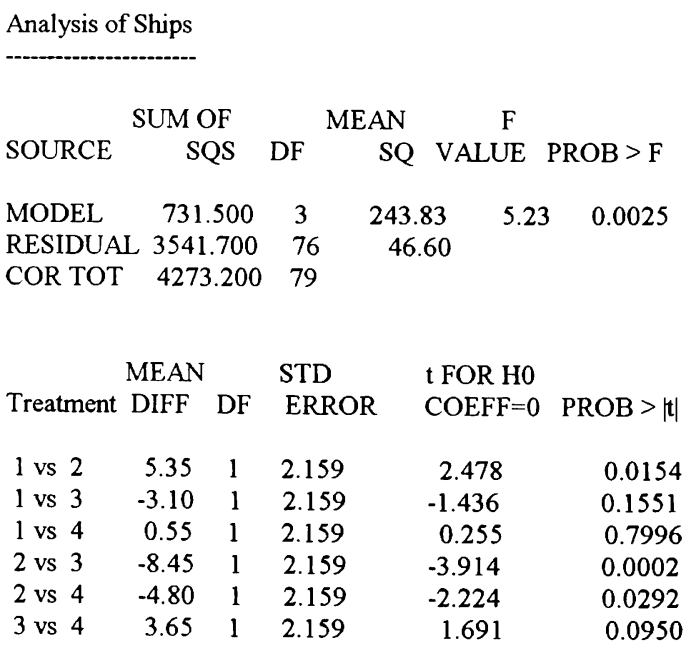

The ANOVA table gives us an $\mathrm{F}$ value of 5.23 , which is significant at a $(1-.0025)$ or $99.75 \%$ level of confidence. So this leads us to reject the null hypothesis that the four mean ships are the same. However, what we really want to know is which means are different, and which one is best. This can be figured by examining the last rows of the ANOVA sheet where a Least Significant 
Difference test is performed. At a $90 \%$ confidence level $(\alpha=.1)$, we can draw the conclusions outlined below:

- Mean ships for Design 1 are statistically higher than Design 2, but not significantly different than Designs 3 and 4.

- Mean ships for Design 2 are also statistically lower than Designs 3 and 4.

- Mean ships for Design 3 are slightly higher than Design 4 (90.5\% confidence).

Therefore, we conclude with $90 \%$ confidence than Design Alternative 2 produces the least amount of ships, and is the worst of the four alternatives. Design Alternative 3 is probably the best strategy of job assignment, but more testing would have to be done to distinguish it from Design Alternative 1.

The results provided by the SLAM II simulation can be judged for reasonableness from a qualitative standpoint as well. It makes sense that design alternative 3 was the most efficient of the alternatives, since it was based on carefully collected labor point data. This data is calculated by the industrial engineer and tells the manager how many hours a shift each machine must be run in order to produce enough product to meet schedules.

Design alternative 2 , on the other hand, was created in a less mathematical way. It is the current arrangement used in the area during the first shift. It makes sense that it is least efficient in terms of output, since it was constructed to balance out the effects of high absenteeism, feuding operators, and other unique managerial factors, rather than maximizing output alone.

One surprise to the researchers was the mediocre performance of design alternative 4 . In this arrangement, all operators were considered to be fully cross-trained, and could rush to any job that needed an operator. Before performing the simulation runs, we expected this alternative to perform the best. However, since there are more machines than operators, machine interference still occurs. In the model, no particular priority was given to bottleneck operations. In the real fab, we would rather an operator wait around idle so he could immediately service a bottleneck machine when necessary. This is better than attending to a nonbottleneck machine and leaving the bottleneck machine idle for any period of time. Design alternative 3 recognizes bottlenecks and deals with them more efficiently.

\section{FUTURE WORK}

The work done to date with the SLAM II model has encouraged the management of the semiconductor facility to expand the model of the entire fab to represent operator-machine assignments. A model from the package MANSIM is being revised to represent operator allocation in the photolithography room, the acid etch room, and thin film deposition areas. Initial work shows that including current operator assignments results in a much more accurate simulation of the day-to-day operations of the facility. The next step is to test possible improved arrangements.

Initial attempts have also been made to test analytical queueing network models of similar systems. These were created and executed much more quickly than the simulation models, but generated less accurate results.

The usefulness of simulation in evaluating operator-machine assignments could also be greatly increased by combining it with mathematical optimization techniques. Integer programming models could be developed to generate test alternatives.

\section{CONCLUSION}

While many approaches exist for solving the operatormachine assignment problem, use of simulation allows the greatest flexibility for unique manufacturing environments. By making use of the RESOURCE block provided in SLAM II, many combinations of operators and machines can be tested to determine a suitable arrangement. This method is not limited to our test model of a rather complex acid etch facility, but could be used in a variety of manufacturing environments. Any facility that has more machines than operators could represent the operators as resources rather than servers. Test alternatives can be quickly generated and analyzed.

The general method of assigning operators to groups of machinery can also be applied in newer dynamic simulation languages. Windows based packages can be altered and tested even more quickly than SLAM II models.

Because this approach is not a mathematical algorithm or expert system, it is limited in the number of alternatives that could be realistically compared. A manager would not want to generate every possible operator-workstation combination. However, this restriction is not critical in a real manufacturing environment. While the number of possible assignment combinations may be large, the number of feasible combinations is much more manageable. A manager can use simulation to test a small number of arrangements he knows to be feasible with respect to the 
constraints of operator cross-training, aptitudes, and relative ability of the operators to work together. As we found alternatives that did not perform as expected, the manager may also be surprised to find out which alternative is most productive. By using a model to test expectations, the manager could save money by not overtraining or over-hiring.

Implementation of this method would provide facilities with a way to evaluate the job assignments they have relied on in the past, and could encourage them to develop a standard arrangement across all shifts. A simulation model that included only the most important elements of the real system could be developed fairly quickly using machine processing data and work measurement standards for the operating personnel. Manufacturing plants with existing simulation models could adapt them to study operator-machine assignments.

Further work could be done to incorporate this evaluative tool with a mathematical method that would truly optimize the operator-machine assignment in relation to the constraints understood by management.

\section{ACKNOWLEDGMENTS}

The authors wish to thank Tyrone Edens, Dick Weddell, Bill Bradley, Fred Blessinger and Scott Schuster of Delco Electronics for their support and assistance in data collection. We also appreciate the efforts of the anonymous appraisers whose comments led to significant improvements in the final paper.

\section{REFERENCES}

Jackman, John and Eric Johnson. 1993. The Role of Queueing Network Models in Performance Evaluation of Manufacturing Systems. Journal of the Operational Research Society. 44(8): 797 807.

Lutz, C.M, K.R. Davis, and C.F. Turner III. 1994. Development of Operator Assignment Schedules: A DSS Approach. Omega, International Journal of Management Science. 22(2): $\quad 57-67$.

Pritsker, A. Alan B. 1995. Introduction to Simulation and SLAM II.

Stafford, Jr. Edward F. 1988. An Optimal Solution Technique for the Operator-Machine Assignment Problem. Production and Inventory Management Journal. 29(3): 25 - 29.

Wittrock, Robert J. 1992. Notes: Operator Assignment and the Parametric Preflow Algorithm. Management Science. 38(9): 1354 1359.

\section{AUTHOR BIOGRAPHIES}

Lynn S. Lachenmaier is a graduate research assistant at Arizona State University, where she is working toward her MSE degree in industrial engineering. Her graduate work is being funded by Delco Electronics, Kokomo, IN, for whom she has worked one year as a manufacturing supervisor. She graduated from Purdue University in 1994 (BSIM).

Jeffery K. Cochran is Associate Professor of Industrial \& Management Systems Engineering at Arizona State University, where he is also a Director of the Systems Simulations Laboratory. Dr. Cochran was educated at Purdue University (BSE 73, MSNE 76, MSIE 82, PhD 84). His industrial experience includes positions at Battelle Northwest Laboratory, Los Alamos National Laboratory, and NASA's Laboratory for Applications of Remote Sensing. Professor Cochran has directed research projects for the National Science Foundation's I/UCRC for Manufacturing and Automation, the National Institute of Standards and Technology, and the Institute for Manufacturing and Automation Research. His primary research interests are simulation modeling and analysis, computer integrated manufacturing, operations research, and artificial intelligence. Dr. Cochran is the author of over fifty scholarly publications, and is a Professional Engineer. 